\title{
Efficacy of the 7-chloro-4-(3-hydroxy-benzilidenehydrazo) quinoline derivative against infection caused by Leishmania amazonensis
}

\author{
Luciana Maria Ribeiro Antinarelli[1],[2], Marcus Vinicius Nora de Souza ${ }^{[3]}$, \\ Eduardo Antonio Ferraz Coelho[ ${ }^{[2],[4]}$, Wallace Pacienza Lima ${ }^{[5],[6]}$ \\ and Elaine Soares Coimbra ${ }^{[1]}$
}

\begin{abstract}
[1]. Universidade Federal de Juiz de Fora, Campus Universitário, Departamento de Parasitologia, Microbiologia e Imunologia, Juiz de Fora, MG, Brasil.
[2]. Universidade Federal de Minas Gerais, Faculdade de Medicina, Programa de Pós-Graduação em Ciências da Saúde: Infectologia e Medicina Tropical, Belo Horizonte, MG, Brasil.

[3]. Fundação Oswaldo Cruz, Instituto de Tecnologia em Fármacos-FarManguinhos, Rio de Janeiro, RJ, Brasil.

[4]. Universidade Federal de Minas Gerais, Colégio Técnico, Departamento de Patologia Clínica, Belo Horizonte, MG, Brasil.

[5]. Universidade Federal do Rio de Janeiro, Instituto de Biofísica Carlos Chagas Filho, Laboratório de Imunofarmacologia, Rio de Janeiro,RJ, Brasil.
\end{abstract}

[6]. Universidade do Grande Rio, Escola de Ciências da Saúde, Duque de Caxias, RJ, Brasil.

\begin{abstract}
Introduction: The drugs currently available for leishmaniasis treatment have major limitations. Methods: In vitro and in vivo studies were performed to evaluate the effect of a quinoline derivative, Hydraqui (7-chloro-4-(3-hydroxy-benzilidenehydrazo)quinoline, against Leishmania amazonensis. In silico analyses of absorption, distribution, metabolism, excretion, and toxicity (ADMET) parameters were performed. Results: Hydraqui showed significant in vitro anti-amastigote activity. Also, Hydraqui-treated mice exhibited high efficacy in lesion size (48.3\%) and parasitic load (93.8\%) reduction, did not cause hepatic and renal toxicity, and showed appropriate ADMET properties. Conclusions: Hydraqui presents a set of satisfactory criteria for its application as an antileishmanial agent.
\end{abstract}

Keywords: Leishmania amazonensis. Quinoline. Chemotherapy. Cutaneous leishmaniasis. Toxicity.

Leishmaniasis is a parasitic infection caused by over 20 species of protozoa of the genus Leishmania. It is characterized by distinct clinical presentation and is dependent on the tropism of the infecting species fort issues such as the skin, mucous membranes, or visceral organs ${ }^{1}$. An estimated 14 million people have been infected across 98 countries $^{2}$. Cutaneous leishmaniasis (CL) presents a high incidence, with an estimated 1-1.5 million new cases occurring worldwide each year. The most recent report estimated 0.3 million new cases of visceral leishmaniasis, the most lethal form of the disease, resulting in over 20,000 deaths each year ${ }^{1}$.

\footnotetext{
Corresponding author: Dra. Elaine Soares Coimbra.

e-mail: elaine.coimbra@ufjf.edu.br

(D) https://orcid.org/0000-0003-1005-278X

Received 27 February 2020

Accepted 14 April 2020
}

Depending on the Leishmania species and the host immune response, CL manifests with distinct clinical and pathological features ${ }^{2}$. Localized cutaneous leishmaniasis is the most prevalent clinical form, characterized by the presence of single or multiple ulcerating lesions, which appear at the site of inoculation by the insect vector ${ }^{2}$. Mucosal leishmaniasis has a more severe manifestation marked by the development of disfiguring lesions in mucosal membranes of the naso-oropharyngeal cavity and is clinically characterized by an intense inflammatory response, tissue damage, and low parasite load ${ }^{2,3}$. Diffuse cutaneous leishmaniasis, a non-ulcerative form of the disease, is accompanied by an immune response triggered by damaged cells, intense parasitism in the lesions, and poor response to chemotherapy ${ }^{2,3}$.

Currently, the most effective drugs used to treat leishmaniasis are pentavalent antimonial-derived products. Amphotericin B deoxycholate and its liposomal formulations, pentamidine and paromomycin, are recommended as alternative drugs. However, 
all available therapeutic options present limitations in terms of long treatment time, intravenous route, systemic toxicity, high cost, therapeutic failure, disease relapse, and/or emerging parasite resistance ${ }^{4,5}$. Miltefosine is still the only approved oral therapeutic option; however, frequent relapse cases, risk of drug-resistant parasites, and varying cure rates against CL in Central and South American countries have led to severe restrictions on the application of this therapy ${ }^{5}$.

Quinoline derivatives have distinct pharmacological applications, including the treatment of bacterial, viral, and protozoa infections. Moreover, it exhibits anti-inflammatory activity and can be used to treat cancer ${ }^{6}$. Several studies have explored this class of scaffolds as promising alternatives for the development of new and selective antileishmanial agents, and some molecules have been identified ${ }^{7-8}$. Consequently, the current study involving this quinoline derivative class of compounds represents a promising strategy to identify new and potent antileishmanial compounds.

In the previous study, we demonstrated the promising in vitro antileishmanial effects of a series of derivatives of 7-chloro-4quinolinyl and hydrazine groups ${ }^{9}$. Among the evaluated compounds, the majority had low toxicity against macrophages and showed significant activity against the promastigotes of four Leishmania species and intra-macrophage amastigotes of L. braziliensis $\left(\mathrm{IC}_{50}\right.$ $<1 \mu \mathrm{M})$. Continuing our investigation with this series of compounds, in this study, we evaluated the in vitro and in vivo effect of 7-chloro4-(3-hydroxy-benzilidenehydrazo)quinoline derivative, Hydraqui, the most effective molecule identified, against L. amazonensis infection for the first time.

For in vitro analysis of anti-amastigote activity, peritoneal macrophages were allowed to adhere to coverslips placed in 24well culture plates for $16 \mathrm{~h}$. Then, L. amazonensis (IFLA/BR/67/ PH8) stationary promastigotes were added to the wells, and the cultures were incubated for $4 \mathrm{~h}$ at $33{ }^{\circ} \mathrm{C}$ in $5 \% \mathrm{CO}_{2}$. The infected macrophages were treated with Hydraqui $(5-100 \mu \mathrm{M})$ for $72 \mathrm{~h}$ at $33{ }^{\circ} \mathrm{C}$ in $5 \% \mathrm{CO}_{2}$ followed by Giemsa staining. The results were evaluated by counting the intracellular amastigotes present in the infected macrophages. $\mathrm{IC}_{50}$ values were obtained of three independent experiments by using the Probit program. Hydraqui was previously synthesized by our research group and characterized using spectroscopic and spectrometric methods ${ }^{9}$. Amphotericin B (AmpB; Cristália, SP, Brazil; 0.06-1.0 $\mu \mathrm{M}$ ) was used as a reference drug.

For the in vivo assay, BALB/c mice (female, $8-10$ weeks of age, 20-25 g of body weight) were obtained from the Biology Research Center at the Federal University of Juiz of Fora (CEUA protocols $\neq 056 / 2013$ and $\neq 046 / 2014)$. Mice were infected with $2 \times 10^{6}$ L. amazonensis-GFP stationary-phase promastigotes. After 7 days of infection, mice were divided into three groups ( 5 mice per group): (i) Control group: treated with $10 \mu \mathrm{L}$ of $1 \times$ PBS containing $5 \%$ DMSO; (ii) AmpB group: treated with $10 \mu \mathrm{L}$ of $1 \times$ PBS containing $250 \mu \mathrm{g} / \mathrm{Kg} \mathrm{AmpB}$; and (iii) Hydraqui group: treated with $10 \mu \mathrm{L}$ of $1 \times$ PBS containing $100 \mu \mathrm{g} / \mathrm{Kg}$ Hydraqui. AmpB and Hydraqui were diluted in a solution of $1 \times \mathrm{PBS}$ containing $5 \%$ DMSO. Mice were treated twice a week and a total of eight doses were administered subcutaneously at the site of infection. Lesion development was monitored before each treatment using a dial caliper (Digimess, SP,
Brazil) ${ }^{7}$. To evaluate the treatment efficacy, mice were euthanized three days after the final treatment ( 35 days post-infection) and the number of viable parasites in the ears of each mouse was estimated using the limiting dilution assay ${ }^{7}$. The parasite burden was measured using fluorometry, as previously described ${ }^{8}$.

For systemic toxicity analysis, sera samples were collected at the end of the experiment, and the levels of alanine aminotransferase (ALT), aspartate transaminase (AST), gamma-glutamyl transpeptidase (GGT), and creatinine were analyzed using laboratory colorimetric kits (Labtest Diagnostica ${ }^{\circledR}$, Belo Horizonte, Minas Gerais, Brazil) ${ }^{7}$. In addition, the weight of each animal was monitored before, during, and after the treatment.

For in silico prediction analysis of drug-likeness, the theoretical properties of absorption, distribution, metabolism, excretion, and toxicity (ADMET) of Hydraqui were evaluated using the admetSAR tool, and the Lipinski's rule of five was calculated using the Molsoft in silico software ${ }^{10}$.

Data were statistically analyzed using the One-way-ANOVA followed by Dunnett's test to compare the treated groups to the negative controls using the Prism 5 software (GraphPad Software, San Diego, CA, USA).

The present study was the first of its kind to demonstrate the in vitro and in vivo activity of a 7-chloroquinoline derivative, Hydraqui, against $L$. amazonensis, a species which presents a significant clinical challenge due to its diverse manifestations during CL, and in some cases, of visceral leishmaniasis in humans, as well its frequent association with poor response to or failure of treatment ${ }^{2}$.

The results of the current study showed that Hydraqui displays significant in vitro activity during treatment of infected macrophages (Figure1A and B). Evaluation of the degree of infection showed that the infection rates were $58.37 \%, 50.33 \%, 61.87 \%, 32.28 \%$, and $16.81 \%$ (Figure 1A), and the number of recovered amastigotes were $3.82,3.28,3.25,1.06$, and 0.46 (Figure 1B) following treatment with $5,10,25,50$, and $100 \mu \mathrm{M}$ of Hydraqui, respectively. The negative control (infected, but untreated macrophages) showed an infection rate of $79.1 \%$, while the number of amastigotes was 6.07 . Based on these results, the $\mathrm{IC}_{50}$ value for Hydraqui was $7.32 \mu \mathrm{M}$, whereas the $\mathrm{IC}_{50}$ for $\mathrm{AmpB}$ was $0.07 \mu \mathrm{M}$.

In this study, the subcutaneous route of treatment of L. amazonensis-infected mice at the infection site was chosen to investigate the in vivo therapeutic effects of Hydraqui. The World Health Organization recommends the inclusion of intralesional treatment among the acceptable therapeutic alternatives, mainly in countries in Central and South America, where the spontaneous cure rate is relatively low for $\mathrm{CL}^{11}$. Local treatment of CL offers several advantages over systemic therapy, including the use of lower drug doses, easy administration, improved safety, non-invasive routes, better patient compliance, fewer adverse effects, and costeffectiveness ${ }^{11}$.

The in vivo therapeutic action of Hydraqui was evaluated by measuring the lesion size and estimating the parasitic load in the infected ears of mice (Figure 2A-D). Our results showed that the progression of lesions was significantly lower after administration of $100 \mu \mathrm{g} / \mathrm{kg}$ of Hydraqui compared to that in the control group 


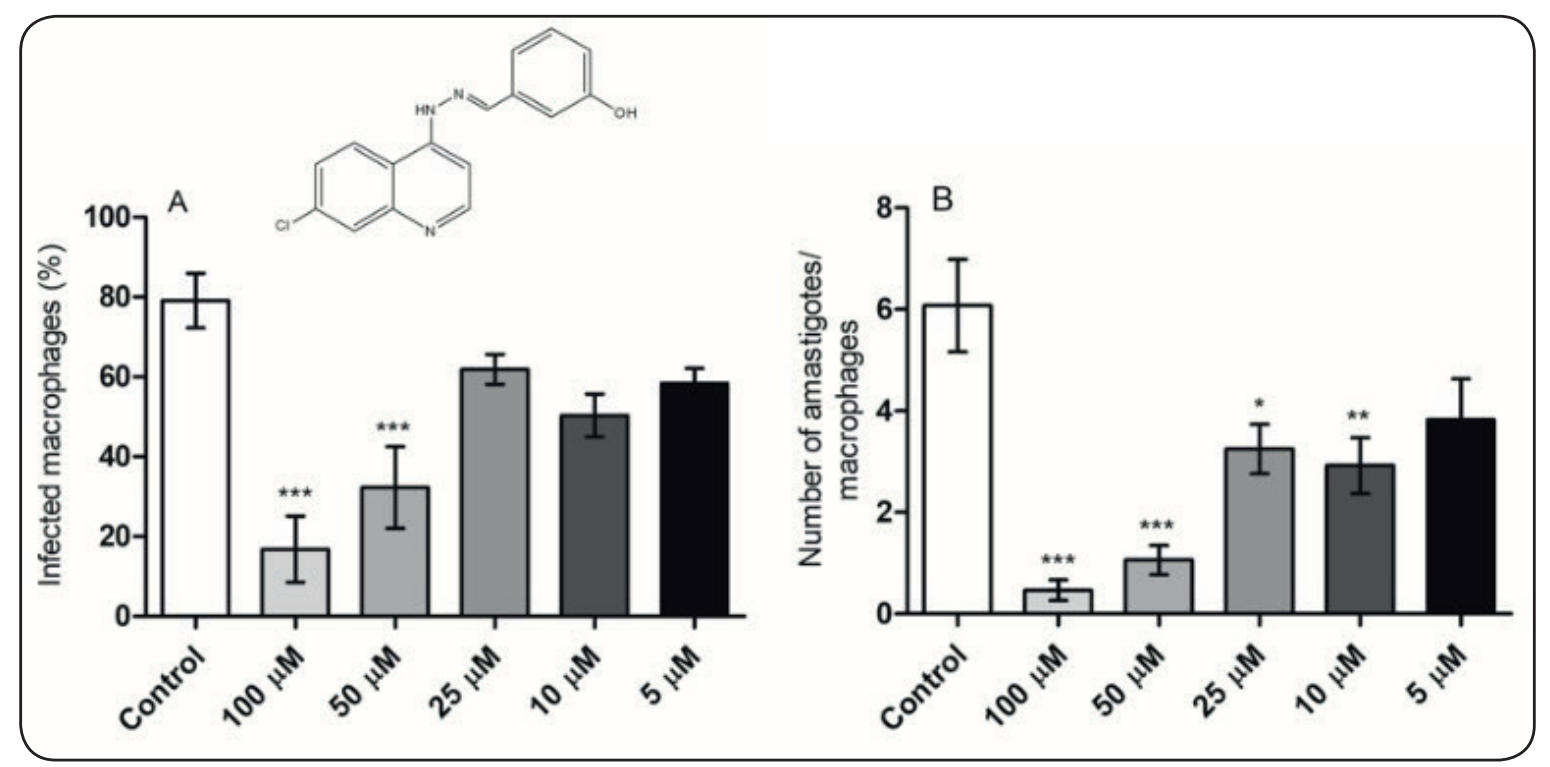

FIGURE 1: Effect of Hydraqui in the treatment of $L$. amazonensis-infected macrophages. Murine macrophages were infected with $L$. amazonensis and treated with the indicated concentrations of Hydraqui for $72 \mathrm{~h}$ at $33^{\circ} \mathrm{C} / 5 \% \mathrm{CO}_{2}$. After the cells were stained with Giemsa, the percentage of infection (A) and the number of recovered amastigotes (B) were calculated by counting 200 cells, in duplicate. Data were expressed as the means of three independent experiments, which were performed in duplicate. ${ }^{* * *} P<0.001$; ${ }^{* *} P<0.01$, and ${ }^{*} P<0.1$ (compared with control group).

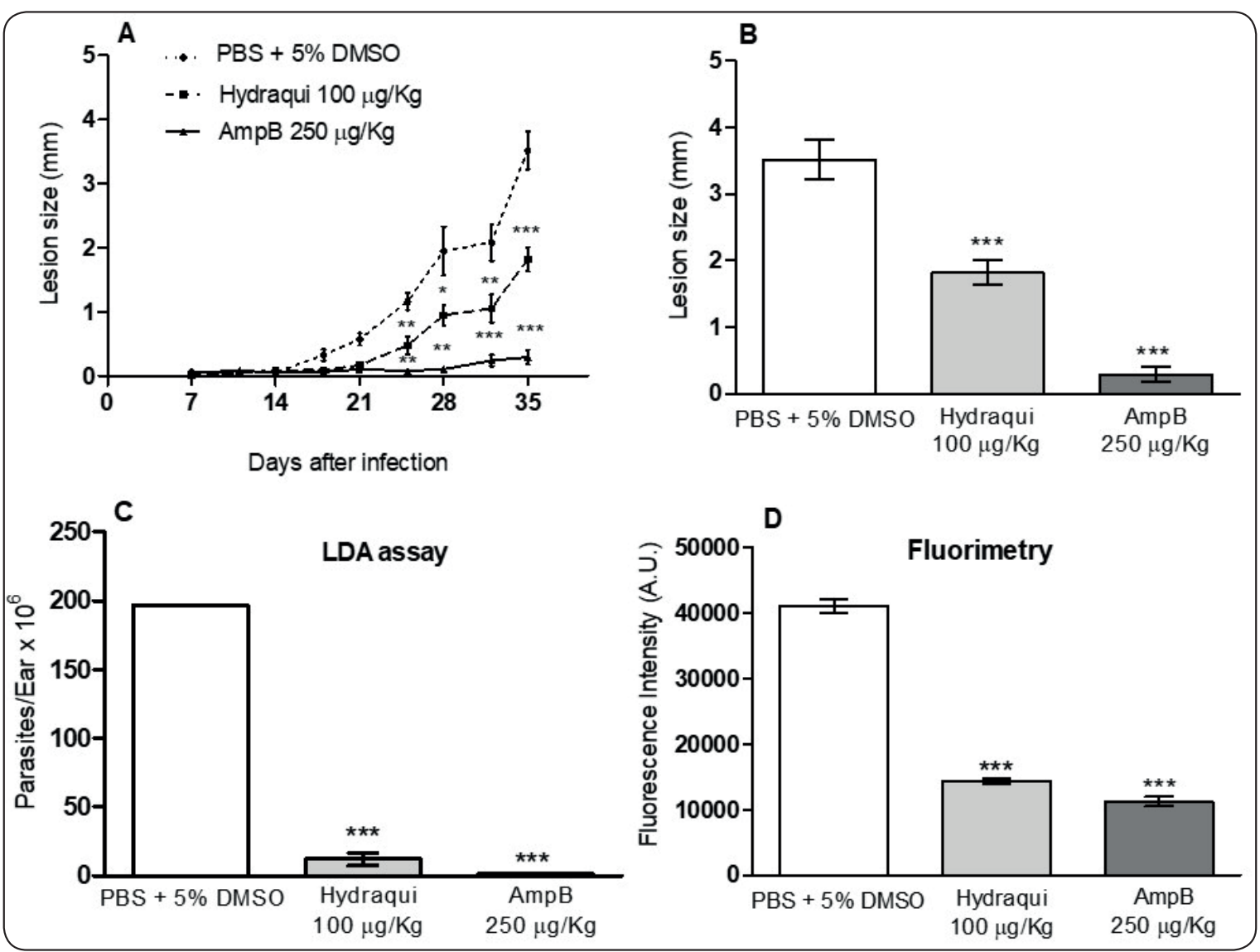

FIGURE 2: Efficacy of Hydraqui in the treatment of $L$. amazonensis-infected mice. The effect of Hydraqui treatment on lesion size was evaluated by measuring the lesion with a dial caliper twice a week until the end of the treatment (A). Lesion size at the end of the treatment (35 days post-infection) (B). Evaluation of the parasite load in the infected tissues 35 days post-infection using limiting dilution analysis (LDA) (C), and by fluorometric assay (D). Fluorometric values are expressed as specific fluorescence units (FU) and are corrected for background uninfected ear values. ${ }^{* * *} P<0.001$, ${ }^{* *} P<0.01$, and ${ }^{*} P<0.1$ (compared with control group). 
(Figure 2A). Lesion growth was significantly reduced (48.3\%) in the Hydraqui group compared with the control group (Figure 2B). Treatment with AmpB (at the highest concentration tested) also proved to be effective in controlling lesion growth. Quantification of the parasitic load using the limiting dilution assay showed that the parasitic load was $93.8 \%$ lower in the experimental group treated with Hydraqui than in the control group (Figure 2C). Indirect quantification of the parasitic burden using fluorometry revealed that the parasitic load was $65.0 \%$ lower in the experimental group treated with Hydraqui than in the control group (Figure 2D). Significant reduction in the parasitic load was also observed in the AmpB group (Figure 2C and 2D). These findings are in agreement with the results of other studies in the literature, which demonstrated that the subcutaneous route of administration is safe and can achieve the antileishmanial effect of 7-chloroquinoline derivatives in vivo ${ }^{7,8}$.

It is interesting to note that Hydraqui did not show any antileishmanial activity against the promastigotes of L. amazonensis ${ }^{9}$. However, in the present study, Hydraqui was effective in vitro against the intracellular forms $\left(\mathrm{IC}_{50} \leq 10 \mu \mathrm{M}\right)$ and showed significant in vivo reductions in lesion size and parasitic load. Stage-specific variations in activities have been widely reported for Leishmania species in vitro and in vivo, but the reasons are not well understood. Several factors may contribute to the in vitro and in vivo variation in sensitivity, including differences in $\mathrm{pH}$, physicochemical profiles favoring entry of the drug into cells via diffusion across the infected macrophages, and drug metabolism, among others ${ }^{12,13}$. Pentavalent antimony is a classic example, as this antileishmanial drug is more effective against the amastigotes than promastigotes of Leishmania species in in vitro assays and also exhibits strong effects in vivo ${ }^{14}$.

Analysis of the in vivo systemic toxicity of Hydraqui in the infected and treated animals was performed at the end of the treatment by measuring the levels of the serum markers of hepatic damage (AST, ALT, and GGT) and renal toxicity (creatinine). The Hydraqui treatment regimen did not affect these markers, indicating the safety of the treatment via the subcutaneous route. Analysis of the experimental group treated with AmpB revealed an increase in ALT and creatinine levels (Figure 3A and 3D). No significant change in body weight was observed in groups treated

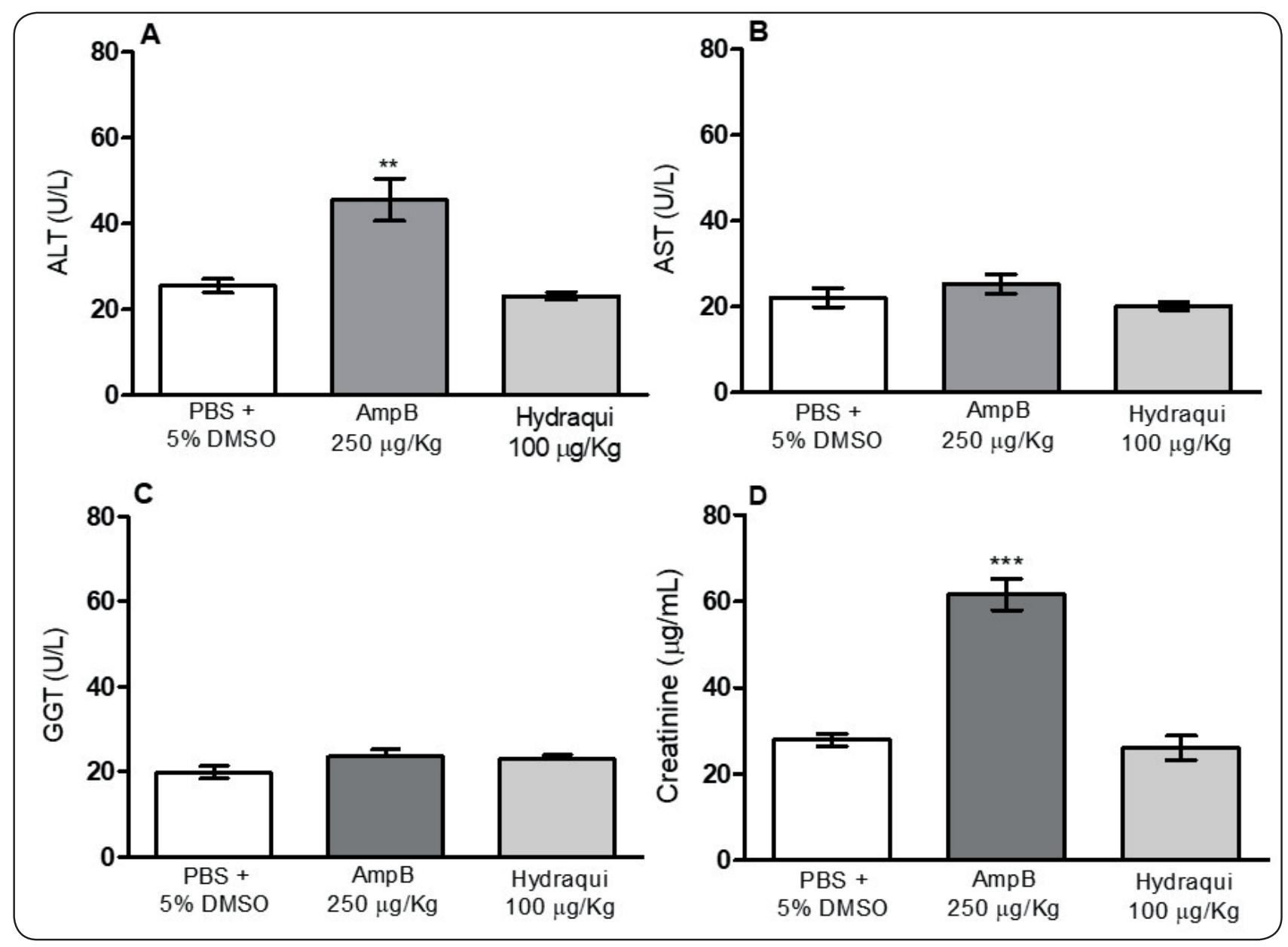

FIGURE 3: Evaluation of systemic toxicity in the treated mice. After $72 \mathrm{~h}$ of the end of the treatment period, serum samples were collected for the determination of the levels of (A) alanine aminotransferase (ALT), (B) aspartate transaminase (AST), (C) gamma-glutamyl transpeptidase (GGT) and (D) creatinine, using colorimetric diagnostic kits. Results are expressed as mean \pm standard deviation, and ${ }^{* * *} P<0.001$ and ${ }^{* *} P<0.01$ represent significant difference compared to the control group. 
with Hydraqui or AmpB compared with the control group (data not shown).

Although the Hydraqui treatment regimen in this study involved the subcutaneous route of administration, theoretical analysis of the physicochemical characteristics (Lipinsky's rule) and ADMET properties were performed to evaluate the safety and bioavailability of this compound following oral administration for future application ${ }^{15}$. Results revealed that Hydraqui presents optimum physicochemical properties, including low molecular weight (297.15), moderate lipophilicity $(\log P=5.15)$, and few functional groups for hydrogen bonding propensity (including four hydrogen-bond acceptors and two donors, data not shown). The calculated ADMET properties showed that Hydraqui has a high probability of human intestinal absorption and Caco-2 cell permeability ( $96.71 \%$ and $55.08 \%$, respectively), suggesting a good profile of oral bioavailability. Regarding its toxicity, this study found that Hydraqui has a class III risk of acute toxicity (as for compounds with median lethal dose $\left[\mathrm{LD}_{50}\right]>500 \mathrm{mg} /$ $\left.\mathrm{kg}^{11}\right)$. A drawback, however, is that Hydraqui demonstrates a high probability of inhibiting the enzymatic activities of CYP450 1A2 and CYP450 3A4, the cytochrome P450 enzymes responsible for drug metabolism (data not shown). These results highlight the potential for administration of Hydraqui through the oral route.

In conclusion, our results indicate that Hydraqui presents a set of satisfactory criteria for its application as an antileishmanial agent. It was highly effective in the treatment of intra-macrophage amastigotes $\left(\mathrm{IC}_{50}\right.$ value $\left.\leq 10 \mu \mathrm{M}\right)$ and demonstrated low toxicity in murine macrophages. Hydraqui-treated mice showed a significant reduction in lesion size and parasitic load, which correlated with the low levels of toxicity markers and appropriate ADMET properties. These findings warrant further studies on Hydraqui to improve its therapeutic efficacy in the treatment of Leishmania spp infections.

\section{ACKNOWLEDGMENTS}

The authors thank the fellowship by CNPq (E.S. Coimbra, L.M.R. Antinarelli, E.A.F. Coelho, and M.V.N. Souza). The authors also thank the Reproduction Biology Center $(C B R)$ of the Federal University of Juiz de Fora for the supply of animals.

\section{FINANCIAL SUPPORT}

This work was financed by Conselho Nacional de Desenvolvimento Científico e Tecnológico (CNPq), Fundação de Amparo à Pesquisa de Minas Gerais (FAPEMIG), and Universidade Federal de Juiz de Fora (UFJF).

\section{AUTHORS' CONTRIBUTION}

LMRA: Conception and design of the study, Acquisition of data, Analysis and interpretation of data, Drafting the article, Final approval of the version to be submitted; MVNS: Conception and design of the study, Synthesis of the compound, Drafting the article; EAFC: Conception and design of the study, Interpretation of data, Drafting the article; WPL: Conception and design of the study, Analysis and interpretation of data; ESC: Conception and design of the study, Interpretation of data, Drafting the article, Final approval of the version to be submitted.

\section{CONFLICTS OF INTEREST}

The authors declare no conflicts of interest.

\section{REFERENCES}

1. World Health Organization, Leishmaniasis, http://www.who.int/topics/ leishmaniasis/en/, 2019, Accessed data: 2 december 2019.

2. Burza S, Croft SL, Boelaert M. Leishmaniasis. Lancet. 2018; 392(10151): 951-70.

3. Scorza BM, Carvalho EM, Wilson ME. Cutaneous Manifestations of Human and Murine Leishmaniasis. Int J Mol Sci. 2017;18(6). pii: E1296.

4. Uliana SRB, Trinconi CT, Coelho AC. Chemotherapy of leishmaniasis: present challenges. Parasitology. 2018;145(4):464-80.

5. Monge-Maillo B, López-Vélez R. Miltefosine for visceral and cutaneous leishmaniasis: drug characteristics and evidence-based treatment recommendations. Clin Infect Dis. 2015;60(9):1398-404.

6. Chu XM, Wang C, Liu W, Liang LL, Gong KK, Zhao CY, et al. Quinoline and quinolone dimers and their biological activities: An overview. Eur $\mathrm{J}$ Med Chem. 2019;161(1):101-17.

7. Antinarelli LMR, de Oliveira Souza I, ZabalaCapriles PV, Gameiro $\mathrm{J}$, Britta EA, Nakamura CV, et al. Antileishmanial activity of a 4-hydrazinoquinoline derivative: Induction of autophagy and apoptosisrelated processes and effectiveness in experimental cutaneous leishmaniasis. Exp Parasitol. 2018;195:78-86.

8. Tavares GSV, Mendonça DVC, Lage DP, Antinarelli LMR, Soyer $\mathrm{TG}$, Senna AJS, et al. In vitro and in vivo antileishmanial activity of a fluoroquinoline derivate against Leishmania infantum and Leishmania amazonensis species. Acta Trop. 2019;191:29-37.

9. Coimbra ES, Antinarelli LM, da Silva AD, Bispo ML, Kaiser CR, de Souza MV. 7-Chloro-4-quinolinyl hydrazones: a promising and potent class of antileishmanial compounds. Chem Biol Drug Des. 2013;81(5):658-65.

10. Cheng F, Li W, Zhou Y, Shen J, Wu Z, Liu G, et al. AdmetSAR: a comprehensive source and free tool for assessment of chemical ADMET properties. J Chem Inf Model. 2012;52(11):3099-105.

11. Ramalho DB, Silva RED, Senna MCR, Moreira HSA, Pedras MJ, Avelar $\mathrm{DM}$, et al. Meglumine antimoniate intralesional infiltration for localized cutaneous leishmaniasis: a single arm, open label, phase II clinical trial. Mem Inst Oswaldo Cruz. 2018;113(9):e180200.

12. De Muylder G, Vanhollebeke B, Caljon G, Wolfe AR, McKerrow J, Dujardin JC. Naloxonazine, an amastigote-specific compound, affects Leishmania parasites through modulation of host-encoded functions. PLoS Negl Trop Dis. 2016;10(12):e0005234.

13. Caridha D, Vesely B, van Bocxlaer K, Arana B, Mowbray CE, Rafati S, et al. Route map for the discovery and pre-clinical development of new drugs and treatments for cutaneous leishmaniasis. Int J Parasitol Drugs Drug Resist. 2019;11:106-17.

14. Eddaikra N, Ait-Oudhia K, Kherrachi I, Oury B, Moulti-Mati F, Benikhlef R, et al. Antimony susceptibility of Leishmania isolates collected over a 30 -year period in Algeria. PLoS Negl Trop Dis. 2018;12(3):e0006310.

15. Lipinski CA, Lombardo F, Dominy BW, Feeney PJ. Experimental and computational approaches to estimate solubility and permeability in drug discovery and development settings. Adv Drug Deliv Rev. 2001;46(1-3):3-26. 